\title{
Analysis of pricing American options on the maximum (minimum) of two risk assets
}

\author{
LISHANG JIANG \\ Institute of Mathematics, Tongji University, People's Republic of China
}

[Received 17 July 2000 and in revised form 7 June 2001]

\begin{abstract}
We use a PDE argument to deal with the mathematical analysis of the valuation of American options on the maximum/minimum of two assets. There are several factors which affect the valuation of options, such as stock price, strike price, the time to expiry, volatilities, the correlation constant, the risk-free interest rate and dividends. The first problem we are concerned with here is what happens to the prices of options if one of these factors is increasing while the others remain fixed. In the second part of this paper, the properties of the optimal exercise boundary of option as free boundary of the parabolic obstacle problem are studied such as monotonicity, convexity and asymptotic behavior.
\end{abstract}

Keywords: American call/put options; parabolic obstacle problem; free boundary; penalized term

In finance a call (put) option is a contract which gives the holder a right to buy (sell) one of the assets, such as stock, foreign currency etc, by a certain date for a certain price. If the option can be exercised only at a certain date, then the contract is known as a European option. If the option can be exercised at any time before a certain date, then the contract is known as an American option.

In the Black-Scholes framework, by a simple arbitrage argument the valuation of the American option is a free boundary problem. Similar to the obstacle problem in mechanics, where the obstacle is the payoff function and the free boundary is the optimal exercise boundary.

In this paper we are concerned with the valuation of American options on the maximum (minimum) of two assets. These are very popular contracts traded in modern financial markets or issued by firms. Many examples can be found in [1-3].

As we know, there are several factors which affect the valuation of American options on the maximum (minimum) of two assets, such as stock prices, strike prices, the time to expiry, volatilities, the correlation constant, the risk-free interest rate and dividends. The first problem considered here is what happens to the prices of the options if one of these factors is increasing while the others remain fixed. For the options of a single asset the same problem was studied in [4: Chapter 7]. For the European option on the maximum (minimum) of two assets, the problem has been solved by Stulz [2] - in this case there is an explicit expression of the price of the option. Unfortunately, we cannot extend his argument to the American case, because here the Black-Scholes model is a variational inequality and the solution cannot be expressed in a closed form. In the first part of this paper, we deal with this problem by using the PDE approach. From the view of PDE the problem is to claim the monotonicity of the solution on variables, initial data and coefficients of equations respectively. In order to prove the monotonicity of the solution, we have to check the sign of related differential expressions of the solution for the variational inequality in which the obstacle and initial data are only Lipschitz continuous. Some results we obtain here are of interest. For example, as is known, the monotonicity of the American option pricing on volatilities in the case of two underlying 
assets is not always true. It depends on their correlation constant. We find a condition and prove that the monotonicity is true if and only if the increments of volatilities satisfy the condition.

The other contribution of this paper is to study the properties of the optimal exercise boundary which is a free boundary of a parabolic obstacle problem. For obstacle problems much is known about the existence, uniqueness and regularity of the solutions. But the obstacle here is a special one in which the payoff function is also initial data of the parabolic obstacle problem. So we can get much deeper information about the properties of the optimal exercise boundary, such as evolution, monotonicity, convexity, asymptotic behavior, etc. Recently Broadie and Detemple published a nice paper [1], in which they also took an interest in the similar problems by using a probability argument.

We start the study of the optimal exercise boundary from a special case of the strike price $E=0$, i.e. the payoff function is $\max \left(S_{1}, S_{2}\right)\left(S_{i}\right.$, the underlying asset $\left.(i=1,2)\right)$, which is the same as the one of an option to exchange one asset for another. This problem was first produced by Margrade [8] for valuing a European option. Rubinstein [9] showed that the American version of this option can be valued similarly to the case of a single asset and solved using the binomial tree method. According to their idea, this problem can be reduced under some transformations to a 1D parabolic obstacle problem with two free boundaries. Some properties of free boundaries are studied, such as monotonicity, asymptotic behavior, etc. Based on all of these results, the evolutions of optimal exercise boundary is studied in the general case $E>0$ using a comparison principle.

The paper is organized as follows. Section 1 outlines the Black-Scholes model of the American call-max (min) option. Section 2 introduces a penalized problem corresponding to the valuation problem of the American call-max ( $\min$ ) option with a smooth approximate payoff function. Sections 3-6 establish the relation between the price of American call (put)-max (min) option and the factors: stock price $S_{1}, S_{2}$, strike price $E$, time to expiration $T$, risk-free interest rate $r$, dividend $q_{1}, q_{2}$, volatility $\sigma_{1}, \sigma_{2}$ and correlation constant $\rho$ respectively. Sections 7 and 8 focus on the properties of the optimal exercise boundary for the American call-max option. We start in Section 7 with the special case of the strike price $E=0$ first. Based on the results of Section 7, the properties of the optimal exercise boundary in the general case of the strike price $E>0$ are dealt with in Section 8.

\section{Model}

Assume the prices of underlying assets $S_{1}, S_{2}$ satisfy the stochastic differential equations

$$
\begin{aligned}
& \mathrm{d} S_{1}=\left(r-q_{1}\right) S_{1} \mathrm{~d} t+\sigma_{1} S_{1} \mathrm{~d} W_{t}^{1} \\
& \mathrm{~d} S_{2}=\left(r-q_{2}\right) S_{2} \mathrm{~d} t+\sigma_{2} S_{2} \mathrm{~d} W_{t}^{2}
\end{aligned}
$$

where $W_{t}^{1}, W_{t}^{2}$ are standard Brownian motions and

$$
\begin{gathered}
E\left(\mathrm{~d} w_{t}^{i}\right)=0, \operatorname{Var}\left(\mathrm{d} W_{t}^{i}\right)=\mathrm{d} t \quad(i=1,2) \\
E\left(\mathrm{~d} w_{t}^{i} \cdot \mathrm{d} w_{t}^{2}\right)=\rho \mathrm{d} t, \quad|\rho|<1
\end{gathered}
$$

where $r$ is constant rate of interest, $q_{i} \geqslant 0$ is dividend rate of asset $i(i=1,2), \sigma_{i}$ is volatility of price of asset $i(i=1,2)$ and $\rho$ is correlation coefficient.

The price $V\left(S_{1}, S_{2}, t\right)$ of an American call option on the maximum of two assets $S_{1}, S_{2}$ (denoted 
American call-max option) satisfies a boundary value problem of PDE as follows:

$$
\begin{array}{rc}
\min \left\{-\frac{\partial V}{\partial t}-L V, V-G\left(S_{1}, S_{2}\right)\right\}=0, & \left(S_{1}, S_{2}\right) \in R_{+} \times R_{+} \\
0 \leqslant t<T \\
V\left(S_{1}, S_{2}, T\right)=G\left(S_{1}, S_{2}\right), & \left(S_{1}, S_{2}\right) \in R_{+} \times R_{+} \\
V\left(0, S_{2}, t\right)=V_{1}^{0}\left(S_{2}, t\right), & S_{2} \in R_{+}, \quad 0 \leqslant t<T \\
V\left(S_{1}, 0, t\right)=V_{2}^{0}\left(S_{1}, t\right), & S_{1} \in R_{+}, \quad 0 \leqslant t<T
\end{array}
$$

where $E$ is the strike price, $T$ is the time to expiry,

$$
\begin{aligned}
L V= & \frac{1}{2}\left(\sigma_{1}^{2} S_{1}^{2} \frac{\partial^{2} V}{\partial S_{1}^{2}}+2 \rho \sigma_{1} \sigma_{2} S_{1} S_{2} \frac{\partial^{2} V}{\partial S_{1} \partial S_{2}}+\sigma_{2}^{2} S_{2}^{2} \frac{\partial^{2} V}{\partial S_{2}^{2}}\right) \\
& +\left(r-q_{1}\right) S_{1} \frac{\partial V}{\partial S_{1}}+\left(r-q_{2}\right) S_{2} \frac{\partial V}{\partial S_{2}}-r V \\
G\left(S_{1}, S_{2}\right)= & \left(\max \left(S_{1}, S_{2}\right)-E\right)^{+}
\end{aligned}
$$

and $V_{i}^{0}(s, t)(i=1,2)$ is a solution of the following problem:

$$
\begin{aligned}
& \min \left\{-\frac{\partial V_{i}^{0}}{\partial t}-\frac{\sigma_{i}^{2}}{2} s^{2} \frac{\partial^{2} V_{i}^{0}}{\partial s^{2}}-\left(r-q_{i}\right) s \frac{\partial V_{i}^{0}}{\partial s}+r V_{i}^{0}, V_{i}^{0}-(s-E)^{+}\right\}=0 \\
& V_{i}^{0}(s, T)=(s-E)^{+} \\
& V_{i}^{0}(0, t)=0 .
\end{aligned}
$$

REMARK 1.1 For other American options on the maximum (minimum) of two assets, the payoff function has a different type as follows:

$$
\begin{array}{ll}
G\left(s_{1}, s_{2}\right)=\left(\min \left(s_{1}, s_{2}\right)-E\right)^{+} & \text {call-min option } \\
G\left(s_{1}, s_{2}\right)=\left(E-\max \left(s_{1}, s_{2}\right)\right)^{+} & \text {put-max option }
\end{array}
$$

and

$$
G\left(s_{1}, s_{2}\right)=\left(E-\min \left(s_{1}, s_{2}\right)\right)^{+} \quad \text { put-min option. }
$$

Then the boundary conditions (1.4), (1.5) have to be changed according to different payoff function.

REMARK 1.2 Equation (1.2) is an obstacle problem of a parabolic equation. The coincidence set and the separate (noncoincidence) set of the obstacle problem are called a stopping region and a continuation region of the option respectively here.

\section{Approximations}

Before starting our proof, we make a transformation

$$
\begin{gathered}
\ln s_{1}=x_{1}, \quad \ln s_{2}=x_{2} \\
T-t=\tau .
\end{gathered}
$$


Then, the American call-max option problem is reduced to a parabolic obstacle problem with constant coefficients as follows:

$$
\begin{aligned}
& \min \left\{\frac{\partial v}{\partial \tau}-\mathcal{L} v, v-g\right\}=0,\left(x_{1}, x_{2}\right) \in R^{2}, 0<\tau \leqslant T \\
& v\left(x_{1}, x_{2}, 0\right)=g\left(x_{1}, x_{2}\right)
\end{aligned}
$$

where

$$
\begin{aligned}
v\left(x_{1}, x_{2}, \tau\right)= & V\left(\mathrm{e}^{x_{1}}, \mathrm{e}^{x_{2}}, T-\tau\right) \\
g\left(x_{1}, x_{2}\right)= & G\left(\mathrm{e}^{x_{1}}, \mathrm{e}^{x_{2}}\right)=\left(\max \left(\mathrm{e}^{x_{1}}, \mathrm{e}^{x_{2}}\right)-E\right)^{+} \\
\mathcal{L} v= & \frac{1}{2}\left[\sigma_{1}^{2} \frac{\partial^{2} v}{\partial x_{1}^{2}}+2 \rho \sigma_{1} \sigma_{2} \frac{\partial^{2} v}{\partial x_{1} \partial x_{2}}+\sigma_{2}^{2} \frac{\partial^{2} v}{\partial x_{2}^{2}}\right] \\
& +\left(r-q_{1}-\frac{\sigma_{1}^{2}}{2}\right) \frac{\partial v}{\partial x_{1}}+\left(r-q_{2}-\frac{\sigma_{2}^{2}}{2}\right) \frac{\partial v}{\partial x_{2}}-r v .
\end{aligned}
$$

As we know, (2.3) is a complementary form of the variational inequality and the payoff function (2.5) is only a Lipschitz continuous function. First we have to consider an approximation of the problem (2.3), (2.4).

Suppose

$$
g_{\varepsilon}\left(x_{1}, x_{2}\right)=\Pi_{\varepsilon}\left(F_{\varepsilon}\left(x_{1}, x_{2}\right)-E\right)
$$

where $\varepsilon>0$ and

$$
\Pi_{\varepsilon}(s)= \begin{cases}s & s \geqslant \varepsilon \\ \nearrow & -\varepsilon \leqslant s \leqslant \varepsilon \\ 0 & s \leqslant-\varepsilon\end{cases}
$$

and

$$
\begin{gathered}
\Pi_{\varepsilon}(s) \in C^{\infty}(R), 1 \geqslant \Pi_{\varepsilon}^{\prime}(s) \geqslant 0, \Pi_{\varepsilon}^{\prime \prime}(s) \geqslant 0, \lim _{\varepsilon \rightarrow 0} \Pi_{\varepsilon}(s)=s^{+} \\
F_{\varepsilon}\left(x_{1}, x_{2}\right)=\frac{\mathrm{e}^{x_{1}}+\mathrm{e}^{x_{2}}}{2}+\frac{\mathrm{e}^{x_{1}}-\mathrm{e}^{x_{2}}}{2} H\left(\frac{\mathrm{e}^{x_{1}}-\mathrm{e}^{x_{2}}}{2 \varepsilon}\right) \\
H(s)=\frac{2}{\pi} \operatorname{arctg}(s) .
\end{gathered}
$$

It is easy to check that

$$
\begin{aligned}
& H^{\prime}(s) \geqslant 0 \\
& 0<2 H^{\prime}(s)+s H^{\prime \prime}(s) \leqslant 4 / \pi \\
& 0 \leqslant 1 \pm\left[H(s)+s H^{\prime}(s)\right] \leqslant 2
\end{aligned}
$$

and

$$
\begin{aligned}
& \lim _{\varepsilon \rightarrow 0} F_{\varepsilon}\left(x_{1}, x_{2}\right)=\max \left(\mathrm{e}^{x_{1}}, \mathrm{e}^{x_{2}}\right) \\
& \lim _{\varepsilon \rightarrow 0} g_{\varepsilon}\left(x_{1}, x_{2}\right)=\lim _{\varepsilon \rightarrow 0} \Pi_{\varepsilon}\left(F_{\varepsilon}\left(x_{1}, x_{2}\right)-E\right)=g\left(x_{1}, x_{2}\right) .
\end{aligned}
$$


Consider an approximation of the complementary form of variational inequality (2.3). In order to do this, we introduce a nonlinear penalized problem as follows:

$$
\frac{\partial v}{\partial \tau}-\mathcal{L} v+\beta_{\delta}(v-g)=0, \quad(\delta>0)
$$

where the penalized term satisfies

$$
\beta_{\delta}(s) \in C^{\infty}(R), \beta_{\delta}(s) \leqslant 0, \beta_{\delta}^{\prime}(s) \geqslant 0, \beta_{\delta}^{\prime \prime}(s) \leqslant 0 .
$$

and

$$
\lim _{\delta \rightarrow 0} \beta_{\delta}(s)= \begin{cases}0 & s>0 \\ -\infty & s<0 .\end{cases}
$$

According to our need in the proof, we will choose the penalized function $\beta_{\delta}(s)$ in the different forms.

Combining the approximation (2.7) and (2.14), we obtain an approximation of the Cauchy problem (2.3), (2.4) as follows:

$$
\begin{aligned}
& \frac{\partial \hat{v}}{\partial \tau}-\mathcal{L} \hat{v}+\beta_{\delta}\left(\hat{v}-g_{\varepsilon}\left(x_{1}, x_{2}\right)\right)=0,\left(x_{1}, x_{2}\right) \in Q_{M} \\
& \left.\hat{v}\right|_{\partial_{p} Q_{M}}=\left.g_{\varepsilon}\left(x_{1}, x_{2}\right)\right|_{\partial_{p}} Q_{M}
\end{aligned}
$$

where $Q_{M}=\left\{-\infty<x_{1} \leqslant M,-\infty<x_{2} \leqslant M ; 0 \leqslant \tau \leqslant T\right\}, M \leqslant \infty, \partial_{p} Q_{M}$ is a parabolic boundary of domain $Q_{M}$, and

$$
\hat{v}\left(x_{1}, x_{2}, \tau\right)=v_{\delta, \varepsilon, M}\left(x_{1}, x_{2}, \tau\right) .
$$

REMARK 2.1 As we know, the solution of $\hat{v}\left(x_{1}, x_{2}, \tau\right)$ of the penalized problem (2.16), (2.17) tends to the solution $v\left(x_{1}, x_{2}, \tau\right)$ of the Cauchy problem (2.3), (2.4) uniformly in any bounded domain $D\left(D \subset Q_{\infty}\right)$ as $\delta \rightarrow 0$ and then $\varepsilon \rightarrow 0, M \rightarrow \infty$, see [5,6].

REMARK 2.2 For American call-main option the approximate payoff function is given in the form

$$
g_{\varepsilon}\left(x_{1}, x_{2}\right)=\Pi_{\varepsilon}\left(\tilde{F}_{\varepsilon}\left(x_{1}, x_{2}\right)-E\right)
$$

where

$$
\tilde{F}_{\varepsilon}\left(x_{1}, x_{2}\right)=\frac{\mathrm{e}^{x_{1}}+\mathrm{e}^{x_{2}}}{2}-\frac{\mathrm{e}^{x_{1}}-\mathrm{e}^{x_{2}}}{2} H\left(\frac{\mathrm{e}^{x_{1}}-\mathrm{e}^{x_{2}}}{2 \varepsilon}\right) .
$$

\section{Effects from change in $s_{1}, s_{2}$ and in $E$}

In this section we assume that

$$
Q_{M}=Q_{\infty}, \text { and } \beta_{\delta}(s) \text { satisfies (2.15). }
$$

By the maximum principle, we have the following lemma. 
LEMMA 3.1

$$
\frac{\partial \hat{v}}{\partial x_{i}} \geqslant 0 \quad \text { in } \quad Q_{\infty}
$$

As its corollary, we have the following theorem.

THEOREM 3.1 If $\bar{S}_{i} \geqslant S_{i},(i=1,2)$, then for the American call-max option

$$
V\left(\bar{s}_{1}, \bar{s}_{2}, t\right) \geqslant V\left(s_{1}, s_{2}, t\right),
$$

where $V\left(s_{1}, s_{2}, t\right)$ is a solution of the problem (1.2)-(1.5).

THEOREM 3.2 If $E_{1} \geqslant E_{2}$, then for the American call-max option

$$
0 \leqslant V\left(s_{1}, s_{2}, t ; E_{2}\right)-V\left(s_{1}, s_{2}, t ; E_{1}\right) \leqslant E_{1}-E_{2} .
$$

Proof. Set $\hat{w}=\hat{v}_{2}-\hat{v}_{1}$, where $\hat{v}_{i}$ is the solution of the penalized problem (2.16), (2.17) corresponding to the strike price $E_{i}(i=1,2)$. In view of

$$
0 \leqslant\left.\hat{w}\right|_{\tau=0}=g_{\varepsilon}^{(2)}-g_{\varepsilon}^{(1)} \leqslant E_{1}-E_{2}
$$

by the maximum principle, we have

$$
\hat{w} \geqslant 0
$$

i.e.

$$
E_{1}-E_{2} \geqslant \hat{v}_{2}\left(x_{1}, x_{2}, \tau\right)-\hat{v}_{1}\left(x_{1}, x_{2}, \tau\right) .
$$

By the same reason, we have

$$
\hat{v}_{2}\left(x_{1}, x_{2}, \tau\right)-\hat{v}_{1}\left(x_{1}, x_{2}, \tau\right) \geqslant 0
$$

so (3.4) is true.

REMARK 3.1 For the American call-min option, it is easy to check that

$$
\left(g_{\varepsilon}\right)_{x_{i}} \geqslant 0
$$

and, therefore, the results of Theorems 3.1 and 3.2 are still true.

\section{Effects from change in $r$ and in $q_{1}, q_{2}$}

In this section we assume that

$$
Q_{M}=Q_{\infty}
$$

and

$$
\beta_{\delta}(s)=-\frac{1}{\delta}(-s)^{+}, \quad(s \in R) .
$$

From the regularities of a solution of the Cauchy problem (2.16), (2.17) with a penalized term (4.1), we know that for any domain $D \subset \subset Q_{\infty}$

$$
\hat{v} \text { and } \nabla \hat{v} \in W_{p}^{2,1}(D), \quad \forall_{p} \in(1, \infty),
$$

where $W_{p}^{2,1}(D)$ is a Sobolev space

$$
W_{p}^{2,1}(D)=\left\{w \mid w, D_{x} w, D_{x}^{2} w, D_{t} w \in L_{p}(D)\right\} .
$$


LEMMA 4.1

$$
\frac{\partial \hat{v}}{\partial x_{i}} \geqslant 0 \quad(i=1,2) .
$$

The proof is similar to that of Lemma 3.1

LEMMA 4.2

$$
\frac{\partial \hat{v}}{\partial x_{1}}+\frac{\partial \hat{v}}{\partial x_{2}}-\hat{v}+\frac{\varepsilon}{2} \geqslant 0
$$

Proof. Setting

$$
w=\frac{\partial \hat{v}}{\partial x_{1}}+\frac{\partial \hat{v}}{\partial x_{2}}-\hat{v}+\frac{\varepsilon}{2}
$$

we have

$$
\begin{aligned}
\frac{\partial w}{\partial \tau}-\mathcal{L} w+\frac{1}{2 \delta}\left(1-S g\left(v-g_{\varepsilon}\right)\right) w & =\frac{1}{2 \delta}\left(1-S g\left(v-g_{\varepsilon}\right)\right)\left(\frac{\varepsilon}{2}+\frac{\partial g_{\varepsilon}}{\partial x_{1}}+\frac{\partial g_{\varepsilon}}{\partial x_{2}}-g_{\varepsilon}\right) \\
\left.w\right|_{\tau=0} & =\frac{\varepsilon}{2}+\frac{\partial g_{\varepsilon}}{\partial x_{1}}+\frac{\partial g_{\varepsilon}}{\partial x_{2}}-g_{\varepsilon}
\end{aligned}
$$

where

$$
S g(s)= \begin{cases}1 & s>0 \\ -1 & s \leqslant 0 .\end{cases}
$$

It is easy to calculate that the function $\varepsilon / 2+\partial g_{\varepsilon} / \partial x_{1}+\partial g_{\varepsilon} / \partial x_{2}-g_{\varepsilon}$ is non-negative. Therefore by using the maximum principle the proof of the lemma is completed.

REMARK 4.1 For the American call-min option, it is easy to see that if $\varepsilon<E \pi / 2$, then the function $\varepsilon / 2+\partial g_{\varepsilon} / \partial x_{1}+\partial g_{\varepsilon} / \partial x_{2}-g_{\varepsilon}$ is still non-negative, and the inequality (4.3) is also true for the American call-min option.

As a corollary of (4.3), we have the following theorem.

THEOREM 4.1 If $r_{1} \geqslant r_{2}$, then for the American call-max option we have

$$
V\left(s_{1}, s_{2}, t ; r_{1}\right) \geqslant V\left(s_{1}, s_{2}, t ; r_{2}\right)
$$

THEOREM 4.2 If $\bar{q}_{i} \geqslant q_{i}(i=1,2)$, then for the American call-max option we have

$$
V\left(s_{1}, s_{2}, t ; \bar{q}_{i}\right) \geqslant V\left(s_{1}, s_{2}, t ; q_{i}\right) .
$$

REMARK 4.2 Thanks to Remark 4.1 the ordering results (4.4), (4.5) are still true for the American call-min option.

\section{Effects from change in expiration time $T$}

In this section we assume

$$
\begin{gathered}
Q_{M}(0<M<\infty) \text {, and that } \beta_{\delta}(s) \text { satisfies (2.15): } \\
\beta_{\delta}(0)=-\frac{1}{\delta} .
\end{gathered}
$$


LEMMA 5.1 If $\delta>0$ is small enough, then

$$
\frac{\partial \hat{v}}{\partial \tau} \geqslant 0
$$

Proof. Due to $\hat{v} \in C^{\infty}\left(Q_{M}\right)$, the function $\omega=\partial \hat{v} / \partial \tau$ satisfies the following problem:

$$
\begin{aligned}
& \frac{\partial \omega}{\partial \tau}-\mathcal{L} \omega+\beta_{\delta}^{\prime}\left(\hat{v}-g_{\varepsilon}\right) \omega=0, \text { in } Q_{M} \\
& \left.\omega\right|_{\tau=0}=\mathcal{L} g_{\varepsilon}-\beta_{\delta}(0) \\
& \left.\omega\right|_{x_{1}=M}=0 \\
& \left.\omega\right|_{x_{2}=M}=0 .
\end{aligned}
$$

By using the maximum principle, the estimate

$$
\mathcal{L} g_{\varepsilon}-\beta_{\varepsilon}(0) \geqslant 0
$$

implies (5.2). According to (2.9), (2.12), a straightforward calculation shows that there exists a constant $\delta_{0}(M)$ such that if $\delta \leqslant \delta_{0}(M)$, the estimate (5.3) holds.

As a corollary of (5.2), we have the following theorem.

THEOREM 5.1 If $T_{1}>T_{2}$, then for the American call-max option

$$
V\left(s_{1}, s_{2}, t ; T_{1}\right) \geqslant V\left(s_{1}, s_{2}, t ; T_{2}\right), \quad\left(t \leqslant T_{2}\right) .
$$

REMARK 5.1 What about the American call-min option? The crucial step is to claim that the inequality (5.3) is still valid. We can do this easily.

\section{Effect from change in volatilities $\sigma_{1}, \sigma_{2}$ and correlation $\rho$}

In this section we assume

$$
Q_{M}=Q_{\infty}
$$

and

$$
\beta_{\delta}(s)=-\frac{1}{\delta}\left[(-s)^{+}\right]^{2} \quad(s \in R) .
$$

LEMMA 6.1 Assume $\bar{\sigma}_{i}=\left(1+\varepsilon_{i}\right) \sigma_{i}, \varepsilon_{i} \geqslant 0(i=1,2)$ and $\left(\varepsilon_{i}, \varepsilon_{2}\right) \in \mathcal{D}$, where the domain $\mathcal{D}$ is enclosed by curves $\Gamma_{1}$ and $\Gamma_{2}$

$$
\begin{cases}\Gamma_{1}=\left\{\left(\varepsilon_{1}, \varepsilon_{2}\right) \mid \varepsilon_{1}=\varphi\left(\varepsilon_{1} / \varepsilon_{2}\right),\right. & \left.0 \leqslant \varepsilon_{1} / \varepsilon_{2} \leqslant \omega_{0}\right\} \\ \Gamma_{2}=\left\{\left(\varepsilon_{1}, \varepsilon_{2}\right) \mid \varepsilon_{2}=\varphi\left(\varepsilon_{2} / \varepsilon_{1}\right),\right. & \left.0 \leqslant \varepsilon_{2} / \varepsilon_{1} \leqslant \omega_{0}\right\}\end{cases}
$$

and

$$
\begin{gathered}
\omega_{0}=\frac{1}{\rho^{2}}\left(1-\sqrt{1-\rho^{2}}\right)^{2} \\
\varphi(\omega)=\frac{-\left(1-\rho^{2}\right)(1+\omega)+\sqrt{\left(1-\rho^{2}\right)^{2}(1+\omega)^{2}+\left(1-\rho^{2}\right)\left[(1+\omega)^{2} \rho^{2}-4 \omega\right]}}{\left(1-\rho^{2}\right)}
\end{gathered}
$$


then we have

$$
\begin{aligned}
\Lambda \hat{v}= & \frac{1}{2}\left(\bar{\sigma}_{1}^{2}-\sigma_{1}^{2}\right) \frac{\partial}{\partial x_{1}}\left(\frac{\partial \hat{v}}{\partial x_{1}}-\hat{v}\right)+\rho\left(\bar{\sigma}_{1} \bar{\sigma}_{2}-\sigma_{1} \sigma_{2}\right) \frac{\partial^{2} \hat{v}}{\partial x_{1} \partial x_{2}} \\
& +\frac{1}{2}\left(\bar{\sigma}_{2}^{2}-\sigma_{2}^{2}\right) \frac{\partial}{\partial x_{2}}\left(\frac{\partial \hat{v}}{\partial x_{2}}-\hat{v}\right) \geqslant 0
\end{aligned}
$$

where $\hat{v}$ is a solution of the penalized problem (2.16), (2.17) with a penalized term (6.1).

Proof. Set $w=\Lambda \hat{v}$, we have

$$
\begin{aligned}
& \frac{\partial w}{\partial \tau}-\mathcal{L} w-\frac{1}{\delta} \Lambda\left[\left(g_{\varepsilon}-\hat{v}\right)^{+}\right]^{2}=0 \\
& \left.w\right|_{\tau=0}=\Lambda g_{\varepsilon} .
\end{aligned}
$$

Computing $\Lambda\left[\left(g_{\varepsilon}-\hat{v}\right)^{+}\right]^{2}$, we obtain that if $\hat{v}>g_{\varepsilon}$,

$$
\Lambda\left[\left(g_{\varepsilon}-\hat{v}\right)^{+}\right]^{2}=0,
$$

and if $\hat{v}<g_{\varepsilon}$,

$$
\Lambda\left[\left(g_{\varepsilon}-\hat{v}\right)^{+}\right]^{2}=I_{+}\left(\left(\hat{v}-g_{\varepsilon}\right)_{x_{1}},\left(\hat{v}-g_{\varepsilon}\right)_{x_{2}}\right)+2\left(\hat{v}-g_{\varepsilon}\right)\left(w-\Lambda g_{\varepsilon}\right)
$$

where $I_{ \pm}\left(\lambda_{1}, \lambda_{2}\right)$ which is a quadratic form is defined as follows:

$$
I_{ \pm}\left(\lambda_{1}, \lambda_{2}\right)=\sigma_{1}^{2}\left(2 \varepsilon_{1}+\varepsilon_{1}^{2}\right) \lambda_{1}^{2}+\sigma_{2}^{2}\left(2 \varepsilon_{2}+\varepsilon_{2}^{2}\right) \lambda_{2}^{2} \pm 2 \rho \sigma_{1} \sigma_{2}\left(\left(\varepsilon_{1}+\varepsilon_{2}\right)+\varepsilon_{1} \varepsilon_{2}\right) \lambda_{1} \lambda_{2} .
$$

Thus

$$
I_{ \pm}\left(\lambda_{1}, \lambda_{2}\right) \geqslant 0, \quad \forall \lambda_{1}, \lambda_{2} \in R
$$

iff

$$
\Delta=-\rho^{2}\left(\left(\varepsilon_{1}+\varepsilon_{2}\right)+\varepsilon_{1} \varepsilon_{2}\right)^{2}+\left(2 \varepsilon_{1}+\varepsilon_{1}^{2}\right)\left(2 \varepsilon_{2}+\varepsilon_{2}^{2}\right) \geqslant 0
$$

i.e.

$$
\begin{gathered}
\omega=\varepsilon_{2} / \varepsilon_{1} \\
\left(1-\rho^{2}\right) \varepsilon_{2}^{2}+2\left(1-\rho^{2}\right)(1+\omega) \varepsilon_{2}-\rho^{2}(1+\omega)^{2}+4 \omega \geqslant 0 .
\end{gathered}
$$

Thus if $\left(\varepsilon_{1}, \varepsilon_{2}\right) \in \mathcal{D}$ and $\mathcal{D}$ is defined by (6.2)-(6.4), then (6.10) follows.

Now we turn to check the sign of $\Lambda g_{\varepsilon}$. A straightforward calculation shows

$$
\begin{aligned}
\Lambda g_{\varepsilon}= & \Pi_{\varepsilon}^{\prime \prime}\left(F_{\varepsilon}-E\right) I_{+}\left(F_{\varepsilon x_{1}}, F_{\varepsilon x_{2}}\right) \\
& +\frac{1}{8 \varepsilon} \Pi_{\varepsilon}^{\prime}\left(F_{\varepsilon}-E\right)\left(2 H^{\prime}(s)+\varepsilon H^{\prime \prime}(s)\right) I_{-}\left(\sigma_{1} \mathrm{e}^{x_{1}}, \sigma_{2} \mathrm{e}^{x_{2}}\right),\left(s=\frac{\mathrm{e}^{x_{1}}-\mathrm{e}^{x_{2}}}{2 \varepsilon}\right) .
\end{aligned}
$$

Therefore, according to the assumption (6.2)-(6.4) and the inequalities (2.9), (2.12), we have

$$
\Lambda g_{\varepsilon} \geqslant 0 \text {. }
$$

Combining (6.8)-(6.11) and using the maximum principle, we have

$$
w=\Lambda \hat{v} \geqslant 0 .
$$


THEOREM 6.1 If $\bar{\sigma}_{i}=\left(1+\varepsilon_{i}\right) \sigma_{i}, \varepsilon_{i} \geqslant 0(i=1,2)$ and $\left(\varepsilon_{1}, \varepsilon_{2}\right) \in \mathcal{D}$, where $\mathcal{D}$ satisfies (6.2)-(6.4), then for the American call-max option

$$
V\left(s_{1}, s_{2}, t ; \bar{\sigma}_{1}, \bar{\sigma}_{2}\right) \geqslant V\left(s_{1}, s_{2}, t ; \sigma_{1}, \sigma_{2}\right) .
$$

Proof. Assume $\hat{v}_{1}\left(x_{1}, x_{2}, \tau\right), \hat{v}\left(x_{1}, x_{2}, \tau\right)$ be the solutions of the penalized problem with volatilities $\bar{\sigma}_{1}$ and $\bar{\sigma}_{2}$ and $\sigma_{1}, \sigma_{2}$ respectively, where the penalized term is the function (6.1), then the function $\hat{w}=\hat{v}_{1}-\hat{v}_{2}$ satisfies the following problem:

$$
\begin{aligned}
& \frac{\partial \hat{w}}{\partial \tau}-\mathcal{L}_{\bar{\sigma}} \hat{w}+\beta^{\prime}(\xi) w=\Lambda \hat{v}_{2} \\
& \left.\hat{w}\right|_{\tau=0}=0
\end{aligned}
$$

where $\mathcal{L}_{\bar{\sigma}}$ is the differential operator $\mathcal{L}$ corresponding to volatilities $\bar{\sigma}_{1}$ and $\bar{\sigma}_{2}$.

By using the maximum principle we have

$$
\hat{w} \geqslant 0
$$

according to the result of Lemma 6.1.

Taking the limit as $\delta \rightarrow 0$ and $\varepsilon \rightarrow 0$, the inequality (6.12) holds.

REMARK 6.1 What about the American call-min option? We check the proof of Lemma 6.1. Under the assumption (6.2)-(6.4), the inequality (6.10) still holds. But in this case,

$$
\begin{aligned}
\Lambda g_{\varepsilon}=\Lambda \Pi_{\varepsilon}\left(\tilde{F}_{\varepsilon}-E\right)= & \Pi_{\varepsilon}^{\prime \prime}\left(\tilde{F}_{\varepsilon}-E\right) I_{+}\left(\tilde{F}_{\varepsilon x_{1}}, \tilde{F}_{\varepsilon x_{2}}\right) \\
& -\frac{1}{8 \varepsilon} \Pi_{\varepsilon}^{\prime}\left(\tilde{F}_{\varepsilon}-E\right)\left(2 H^{\prime}(s)+s H^{\prime \prime}(s)\right) I_{-}\left(\sigma_{1} \mathrm{e}^{x_{1}}, \sigma_{2} \mathrm{e}^{x_{2}}\right) .
\end{aligned}
$$

Thus for all $\left(x_{1}, x_{2}\right) \in R^{2}$ the sign of $\Lambda g_{\varepsilon}$ is not constant. That means the result of Theorem 6.1 is not true for the American call-min option!

But for the American put option, the situation is opposite. Theorem 6.1 holds for the American put-min option, but it is false for the American put-max option.

REMARK 6.2 It is clear that the domain $\mathcal{D}$ in the assumption (6.2) depends on the correlation constant $\rho$. Denoting $\mathcal{D}$ by $\mathcal{D}_{\rho}$, we have

(1) $\mathcal{D}_{\rho_{1}} \supset \mathcal{D}_{\rho_{2}}$ if $\left|\rho_{1}\right|<\left|\rho_{2}\right|$,

(2) $\mathcal{D}_{0}=R^{+} \times R^{+}$,

(3) $\mathcal{D}_{\rho} \rightarrow \mathcal{D}_{1}=\left\{\left(\varepsilon_{1}, \varepsilon_{2}\right) \mid \varepsilon_{1}=\varepsilon_{2}, \forall \varepsilon_{i} \in R^{+}, i=1,2\right\}$ as $|\rho| \rightarrow 1$.

Finally we study the effect of change in correlation $\rho$.

In view of the definitions of $g_{\varepsilon}\left(x_{1}, x_{2}\right)$

$$
\frac{\partial^{2} g_{\varepsilon}}{\partial x_{1} \partial x_{2}}=\left\{\begin{array}{lll}
\Pi_{\varepsilon}^{\prime \prime}\left(F_{\varepsilon}-E\right)\left(F_{\varepsilon}\right)_{x_{1}}\left(F_{\varepsilon}\right)_{x_{2}}+\Pi_{\varepsilon}^{\prime}\left(F_{\varepsilon}-E\right)\left(F_{\varepsilon}\right)_{x_{1} x_{2}} & \text { call-max option } \\
\Pi_{\varepsilon}^{\prime \prime}\left(E-F_{\varepsilon}\right)\left(F_{\varepsilon}\right)_{x_{1}}\left(F_{\varepsilon}\right)_{x_{2}}-\Pi_{\varepsilon}^{\prime}\left(E-F_{\varepsilon}\right)\left(F_{\varepsilon}\right)_{x_{1} x_{2}} & \text { put-max option } \\
\Pi_{\varepsilon}^{\prime \prime}\left(\tilde{F}_{\varepsilon}-E\right)\left(\tilde{F}_{\varepsilon}\right)_{x_{1}}\left(\tilde{F}_{\varepsilon}\right)_{x_{2}}+\Pi_{\varepsilon}^{\prime}\left(\tilde{F}_{\varepsilon}-E\right)\left(\tilde{F}_{\varepsilon}\right)_{x_{1} x_{2}} & \text { call-min option } \\
\Pi_{\varepsilon}^{\prime \prime}\left(E-\tilde{F}_{\varepsilon}\right)\left(\tilde{F}_{\varepsilon}\right)_{x_{1}}\left(\tilde{F}_{\varepsilon}\right)_{x_{2}}-\Pi_{\varepsilon}^{\prime}\left(E-\tilde{F}_{\varepsilon}\right)\left(\tilde{F}_{\varepsilon}\right)_{x_{1} x_{2}} & \text { put-min option. }
\end{array}\right.
$$


TABLE 1

\begin{tabular}{|c|c|c|c|c|c|c|c|c|}
\hline & \multicolumn{4}{|c|}{ Max-option } & \multicolumn{4}{|c|}{ Min-option } \\
\hline & \multicolumn{2}{|c|}{ European } & \multicolumn{2}{|c|}{ American } & \multicolumn{2}{|c|}{ European } & \multicolumn{2}{|c|}{ American } \\
\hline & Call & Put & Call & Put & Call & Put & Call & Put \\
\hline Stock price $s_{1}, s_{2}$ & + & - & + & - & + & - & + & - \\
\hline Strike price $E$ & - & + & - & + & - & + & - & + \\
\hline Time to expiration & $?$ & $?$ & + & + & $?$ & ? & + & + \\
\hline Volatility $\sigma_{1}, \sigma_{2}^{(*)}$ & + & $?$ & + & ? & $?$ & + & $?$ & + \\
\hline Correlation $\rho$ & $?$ & + & $?$ & $? ?$ & + & $?$ & $? ?$ & $?$ \\
\hline Risk-free interest rate $r$ & + & - & + & - & + & - & + & - \\
\hline Dividend $q_{1}, q_{2}$ & - & + & - & + & - & + & - & + \\
\hline
\end{tabular}

${ }^{(*)}$ The increments of $\sigma_{1}$ and $\sigma_{2}$ have to satisfy conditions (6.2)-(6.4).

(?): the conclusion of monotonicity on this variable is not true.

(??): the conclusion of monotonicity on this variable is still unknown.

According to $\left(F_{\varepsilon}\right)_{x_{1} x_{2}} \leqslant 0,\left(\tilde{F}_{\varepsilon}\right)_{x_{1} x_{2}} \geqslant 0$ and (2.9), (2.12) we have for the put-max option and the call-min option

$$
\frac{\partial^{2} g_{\varepsilon}}{\partial x_{1} \partial x_{2}} \geqslant 0
$$

But for the call-max option and the put-min option the sign of $\frac{\partial^{2} g_{\varepsilon}}{\partial x_{1} \partial x_{2}}$ is not constant. As a consequence of the above fact, we have the following theorem.

THEOREM 6.2 If $\bar{\rho} \geqslant \rho$, then for the European put-max option and the European call-min option, the price of option $V_{e}\left(s_{1}, s_{2}, t\right)$ satisfies

$$
V_{E}\left(s_{1}, s_{2}, t ; \bar{\rho}\right) \geqslant V_{E}\left(s_{1}, s_{2}, t ; \rho\right) .
$$

However, this condition is false for the European call-max option and the European put-min option. Whether the result is true for the American put-max option and American call-min option remains an open question.

The results obtained in Sections 3-6 are summarized in Table 1.

\section{Evolution of optimal exercise boundary in the case of $E=0$}

In this section and the next, we confine ourselves to study of the properties of the optimal exercise boundary only for the American call-max option. We believe that for other options the proof is similar, although the results may be different.

We first study an evolution of optimal exercise boundary in the case of $E=0$, and assume $q_{1}+q_{2}>0$ (wlog, $q_{2}>0$ ). In fact, as we know, for the American call option there is not an optimal exercise boundary at all, if $q_{1}=q_{2}=0$.

From (1.2)-(1.5), the price $V\left(s_{1}, s_{2}, t\right)$ of the American call-max option is a solution of the 
following problem:

$$
\begin{aligned}
& \min \left\{\frac{\partial V}{\partial t}-L V, V-\max \left(s_{1}, s_{2}\right)\right\}=0 \\
& \left.V\right|_{t=T}=\max \left(s_{1}, s_{2}\right) \\
& \left.V\right|_{s_{1}=0}=s_{2} \\
& \left.V\right|_{s_{2}=0}=s_{1} .
\end{aligned}
$$

Set

$$
V=s_{2} U
$$

introduce a new variable

$$
\xi=\frac{s_{1}}{s_{2}}
$$

and define

$$
u(\xi, t)=U\left(s_{1}, s_{2}, t\right)
$$

Then we have

$$
\begin{aligned}
& \min \left\{-\frac{\partial u}{\partial t}-L_{0} u, u-\max (\xi, 1)\right\}=0 \\
& \left.u\right|_{t=T}=\max (\xi, 1) \\
& \left.u\right|_{\xi=0}=1
\end{aligned}
$$

where

$$
L_{0} u=\frac{1}{2}\left(\sigma_{1}^{2}-2 \rho \sigma_{1} \sigma_{2}+\sigma_{2}^{2}\right) \xi^{2} \frac{\partial^{2} u}{\partial \xi^{2}}+\left(q_{2}-q_{1}\right) \xi \frac{\partial u}{\partial \xi}-q_{2} u
$$

THEOREM 7.1 A solution $u(\xi, t)$ of problem (7.7)-(7.9) satisfies the following free boundary problem:

$$
\begin{gathered}
-\frac{\partial u}{\partial t}-L_{0} u=0 \quad \xi_{1}(t)<\xi<\xi_{2}(t), \quad 0 \leqslant t<T \\
\left.u\right|_{\xi=\xi_{1}(t)}=1,\left.\frac{\partial u}{\partial \xi}\right|_{\xi=\xi_{1}(t)}=0, \quad 0 \leqslant t<T \\
\left.u\right|_{\xi=\xi_{2}(t)}=\xi_{2}(t),\left.\frac{\partial u}{\partial \xi}\right|_{\xi=\xi_{2}(t)}=1, \quad 0 \leqslant t<T
\end{gathered}
$$

where $\xi_{1}(t), \xi_{2}(t)$ are free boundaries and

$$
\xi_{1}(t) \uparrow, \quad \xi_{2}(t) \downarrow
$$

and

$$
\xi_{1}(T)=\xi_{2}(T)=1
$$


Proof. Assume $\mathcal{C}$ to be a continuation region of the American call-max option $(E=0)$, and $\partial \mathcal{C}$ to be the boundary of $\mathcal{C}$.

First, we claim

$$
\partial_{T} \mathcal{C}=\partial \mathcal{C} \cap\{t=T\}=\operatorname{Point}(1, T) .
$$

In $\mathcal{C}$, as we know, $u(\xi, t)$ is the solution of the problem (7.7)-(7.9). If $(\xi, T) \in \partial_{T} C$ and $\xi \neq 1$, then from (7.7) and the initial data (7.8) we have

$$
\left.\frac{\partial u}{\partial t}\right|_{(\xi, T)}=L_{0}(\max (1, \xi))= \begin{cases}q_{1} \xi & \xi>1 \\ q_{2} & \xi<1\end{cases}
$$

Therefore in the neighborhood of $(\xi, T),(\xi \neq 1)$,

$$
u(x, t)<\max (1, \xi), \quad(x, t) \in \mathcal{C} .
$$

The contradiction shows that (7.16) is true.

Thus there exist two free boundaries which start from $(1, T)$. The property (7.14) is a consequence of the following fact:

$$
\frac{\partial u}{\partial t} \leqslant 0
$$

The proof of (7.18) is same as for Lemma 5.1.

We next study the asymptotic behavior of a solution to the parabolic obstacle problem (7.1)-(7.4) as $T \rightarrow \infty$.

Consider an elliptic obstacle problem as follows.

Find a triple $\left\{\hat{u}_{0}, \xi_{1}^{0}, \xi_{2}^{0}\right\}$ such that

$$
\begin{array}{rlrl}
L_{0} \hat{u}_{0} & =0, & \xi_{1}^{0}<\xi<\xi_{2}^{0} \\
u_{0}\left(\xi_{1}^{0}\right) & =1, & u_{0}^{\prime}\left(\xi_{1}^{0}\right)=0 \\
u_{0}\left(\xi_{2}^{0}\right) & =\xi_{2}^{0}, & & u_{0}^{\prime}\left(\xi_{2}^{0}\right)=1
\end{array}
$$

where $L_{0} u$ is defined by (7.10).

Problem (7.19)-(7.21) is a second-order ordinary differential equation with two free boundary points. It has a solution

$$
\hat{u}=c_{1}\left(\xi / \xi_{2}^{0}\right)^{\alpha_{+}}+c_{2}\left(\xi / \xi_{1}^{0}\right)^{\alpha_{-}}
$$

where $\alpha_{ \pm}$are roots of the following quadratic equation:

$$
\begin{gathered}
\frac{1}{2} \sigma^{2} \alpha(\alpha-1)+\left(q_{2}-q_{1}\right) \alpha-q_{2}=0 \\
\left(\sigma^{2}=\alpha_{1}^{2}-2 \rho \alpha_{1} \alpha_{2}+\alpha_{2}^{2}\right) \quad \alpha_{-}<0, \quad \alpha_{+}>1
\end{gathered}
$$

and

$$
\alpha_{ \pm}=w \pm \theta
$$


where

$$
\begin{gathered}
w=\frac{1}{2}+\frac{1}{\sigma^{2}}\left(q_{1}-q_{2}\right) \\
\theta=\sqrt{\omega^{2}+\frac{2 q_{2}}{\sigma^{2}}}=\sqrt{\frac{1}{4}+\frac{q_{1}}{\sigma^{2}}+\frac{\left(q_{1}-q_{2}\right)^{2}}{\sigma^{4}}} .
\end{gathered}
$$

The coefficients $c_{1}, c_{2}$ and free boundary points $\xi_{1}^{0}, \xi_{2}^{0}$ are defined by boundary conditions (7.20), (7.21). Therefore we get the solution

$$
\begin{aligned}
& \hat{u}_{0}=\frac{\alpha_{-}}{\alpha_{-}-\alpha_{+}} \beta_{-}^{\frac{\alpha_{+}\left(1-\alpha_{-}\right)}{\alpha_{+}-\alpha_{-}}} \beta_{+}^{-\frac{\alpha_{+}\left(1+\alpha_{+}\right)}{\alpha_{+}-\alpha_{-}}} \xi^{\alpha_{+}}+\frac{\alpha_{+}}{\alpha_{+}-\alpha_{-}} \beta_{+}^{\frac{\alpha_{-}\left(\alpha_{+}-1\right)}{\alpha_{+}-\alpha_{-}}} \beta_{-}^{\frac{\alpha_{-}\left(1-\alpha_{-}\right)}{\alpha_{+}-\alpha_{-}}} \xi^{\alpha_{-}} \\
& \xi_{1}^{0}=\beta_{+}^{\frac{1-\alpha_{+}}{\alpha_{+}-\alpha_{-}}} \beta_{-}^{\frac{\alpha_{-}-1}{\alpha_{+}-\alpha_{-}}} \\
& \xi_{2}^{0}=\beta_{+}^{\frac{-\alpha_{+}}{\alpha_{+}-\alpha_{-}}} \beta_{-}^{\frac{\alpha_{-}}{\alpha_{-}-\alpha_{-}}}
\end{aligned}
$$

where $\beta_{ \pm}=\left(\alpha_{ \pm}-1\right) / \alpha_{ \pm}$.

Thus we have proved the following theorem.

THEOREM 7.2 The elliptic obstacle problem

$$
\begin{gathered}
\min \left\{L_{0} u_{0}, u_{0}-\max (1, \xi)\right\}=0 \\
u_{0}(0)=1,
\end{gathered}
$$

has a solution

$$
u_{0}(\xi)= \begin{cases}\xi & \xi \geqslant \xi_{2}^{0} \\ \hat{u}_{0}(\xi) & \xi_{1}^{0} \leqslant \xi \leqslant \xi_{2}^{0} \\ 1 & 0 \leqslant \xi \leqslant \xi_{1}^{0}\end{cases}
$$

where the triple $\left\{\hat{u}_{0}, \xi_{1}^{0}, \xi_{2}^{0}\right\}$ is a solution of the free boundary problem (7.19)-(7.21), which is expressed in (7.25)-(7.27).

Denoting the pricing of the American option and the optimal exercise boundary in the problem (7.11)-(7.13) for the expiration time $T$ by $u(\xi, t ; T)$ and $\xi_{i}(t ; T)$, then we have the following theorem.

THEOREM 7.3 The solution $u_{0}(\xi)$ (7.30) is a limit of the solution $u(\xi, t ; T)$ of the problem (7.7)(7.9) (also (7.11)-(7.13)) as $T \rightarrow \infty$, i.e. $\forall t<T$

$$
\lim _{T \rightarrow \infty} u(\xi, t ; T)=u_{0}(\xi), \quad \xi \in R
$$

and

$$
\lim _{T \rightarrow \infty} \xi_{i}(t ; T)=\xi_{i}^{0}, \quad(i=1,2) .
$$


In order to prove this, we make a transformation $\tau=T-t$ in the problem (7.7)-(7.9) and study the asymptotic behavior of the solution as $\tau \rightarrow \infty$.

The proof of the theorem is standard. We omit it here.

THEOREM 7.4 Let $V\left(s_{1}, s_{2}, t\right)$ be the price of the American call-max option (strike price $E=0$ ), then the optimal exercise boundary consists of two surfaces

$$
s_{1}=\xi_{1}(t) s_{2}
$$

and

$$
s_{1}=\xi_{2}(t) s_{2}
$$

The continuation region is

$$
\left\{\xi_{1}(t)<\frac{s_{1}}{s_{2}}<\xi_{2}(t),\left(s_{1}, s_{2}\right) \in R^{+} \times R^{+}, 0 \leqslant t \leqslant T\right\}
$$

and the stopping region $\sum_{0}=\sum_{0}^{(1)} \cup \sum_{0}^{(2)}$,

$$
\begin{aligned}
& \sum_{0}^{(1)}=\left\{0 \leqslant s_{2} \leqslant s_{1} / \xi_{2}(t), 0 \leqslant t \leqslant T,\left(s_{1}, s_{2}\right) \in R^{+} \times R^{+}\right\} \\
& \sum_{0}^{(2)}=\left\{0 \leqslant s_{1} \leqslant s_{2} \xi_{1}(t), 0 \leqslant t \leqslant T,\left(s_{1}, s_{2}\right) \in R^{+} \times R^{+}\right\}
\end{aligned}
$$

where $\xi_{i}(t)(i=1,2)$ are the free boundaries of problem (7.11)-(7.13). As $T \rightarrow \infty$, the price of perpetual American call-max option (strike price $E=0$ ) is

$$
V_{\infty}\left(s_{1}, s_{2}\right)= \begin{cases}s_{1} & \frac{s_{1}}{s_{2}} \geqslant \xi_{2}^{0} \\ \frac{\alpha_{-}}{\alpha_{-}-\alpha_{+}} \beta_{-}^{\frac{\alpha+\left(1-\alpha_{-}\right)}{\alpha_{+}-\alpha_{-}}} \beta_{+}^{-\frac{\alpha+\left(1+\alpha_{+}\right)}{\alpha_{+}-\alpha_{-}}} s_{1}^{\alpha_{+}} s_{2}^{1-\alpha_{+}} & \\ \quad+\frac{\alpha_{+}}{\alpha_{+}-\alpha_{-}} \beta_{+}^{\frac{\alpha-\left(\alpha_{+}-1\right)}{\alpha_{+}-\alpha_{-}}} \beta_{-}^{\frac{\alpha-\left(1-\alpha_{-}\right)}{\alpha_{+}-\alpha_{-}}} s_{1}^{\alpha_{-}} s_{2}^{1-\alpha_{-}} & \xi_{1}^{0} \leqslant \frac{s_{1}}{s_{2}} \leqslant \xi_{2}^{0} \\ s_{2} & 0 \leqslant \frac{s_{1}}{s_{2}} \leqslant \xi_{1}^{0} .\end{cases}
$$

Theorem 7.4 is the consequence of Theorems 7.2 and 7.3 according to the transformations (7.5), (7.6).

\section{Properties of the optimal exercise boundary}

Let us turn to the American call-max option in the general case $E>0$, and study the properties of the optimal exercise boundary.

Suppose $\sum(t ; E)$ to be the stopping region of the American call-max option, i.e.

$$
\sum(t ; E)=\left\{\left(s_{1}, s_{2}\right) \mid V\left(s_{1}, s_{2}, t\right)=G_{E}\left(s_{1}, s_{2}\right), s_{1} \in R^{+}, s_{2} \in R^{+}\right\}
$$


where $G_{E}\left(s_{1}, s_{2}\right)=\left(\max \left(s_{1}, s_{2}\right)-E\right)^{+}$. The optimal exercise boundary $\Gamma(t ; E)=\Gamma_{1}(t ; E) \cup$ $\Gamma_{2}(t ; E)$, where

$$
\begin{aligned}
& \Gamma_{1}(t ; E)=\partial \sum(t ; E) \cap\left\{s_{1} \geqslant s_{2}\right\} \\
& \Gamma_{2}(t ; E)=\partial \sum(t ; E) \cap\left\{s_{2}>s_{1}\right\} .
\end{aligned}
$$

It is clear that

$$
\sum(T ; E)=\left\{\left(s_{1}, s_{2}\right) \mid L G_{E}\left(s_{1}, s_{2}\right)<0\right\}
$$

In fact, if $\left(s_{1}^{0}, s_{2}^{0}, T\right)$ belongs to the continuation region and $\left.L G_{E}\right|_{\left(s_{1}^{0}, s_{2}^{0}\right)}<0$, then we have

$$
-\left.\frac{\partial V}{\partial t}\right|_{\left(s_{1}^{0}, s_{2}^{0}, T\right)}=\left.L G_{E}\right|_{\left(s_{1}^{0}, s_{2}^{0}\right)}<0
$$

which means that there exists $t_{0}<T$, such that $\left(s_{1}^{0}, s_{2}^{0}, t_{0}\right)$ belongs to the continuity region, and

$$
V\left(s_{1}^{0}, s_{2}^{0}, t_{0}\right)<V\left(s_{1}^{0}, s_{2}^{0}, T\right)=G_{E}\left(s_{1}^{0}, s_{2}^{0}\right)
$$

That is impossible.

Therefore strightforward calculation from (8.3) shows that for the American call-max option the stopping region at expiration time $t=T$ is

$$
\sum(T ; E)=\left\{\left(s_{1}, s_{2}\right) \mid s_{1} \geqslant s_{2}, s_{1} \geqslant \max \left(E, \frac{E r}{q_{1}}\right) \text { and } s_{1} \leqslant s_{2}, s_{2} \geqslant \max \left(E, \frac{E r}{q_{2}}\right)\right\} .
$$

What about the evolution of the optimal exercise boundary (stopping region) as $t<T$ ?

\section{THEOREM 8.1}

$$
\sum\left(t_{2} ; E\right) \supset \sum\left(t_{1} ; E\right) \text { as } t_{2}>t_{1}
$$

i.e. the free boundary is monotonic.

This is a consequence of the estimate (5.2) $\frac{\partial V}{\partial t} \leqslant 0$.

THEOREM 8.2

$$
\sum\left(t ; E_{1}\right) \subset \sum\left(t ; E_{2}\right), \quad\left(E_{1}>E_{2}\right) .
$$

Proof. If this is not true, there exists a point $\left(s_{1}^{0}, s_{2}^{0}\right)$ and time $t_{0}$ such that

$$
\left(s_{1}^{0}, s_{2}^{0}, t_{0}\right) \in \sum\left(t_{0} ; E_{1}\right) \text { but }\left(s_{1}^{0}, s_{2}^{0}, t_{0}\right) \notin \sum\left(t_{0} ; E_{2}\right) .
$$

Then, in view of (3.4), we have

$$
\begin{aligned}
G_{E_{1}}\left(s_{1}^{0}, s_{2}^{0}\right) & =V\left(s_{1}^{0}, s_{2}^{0}, t_{0} ; E_{1}\right) \\
& \geqslant V\left(s_{1}^{0}, s_{2}^{0}, t_{0} ; E_{2}\right)-E_{1}+E_{2} \\
& >G_{E_{2}}\left(s_{1}^{0}, s_{2}^{0}\right)-E_{1}+E_{2}
\end{aligned}
$$


i.e.

$$
\max \left(\max \left(s_{1}^{0}, s_{2}^{0}\right)-E_{1}, 0\right)+E_{1}>\max \left(\max \left(s_{1}^{0}, s_{2}^{0}\right)-E_{2}, 0\right)+E_{2} .
$$

Consequently,

$$
\max \left(s_{1}^{0}, s_{2}^{0}\right)<E_{1}
$$

but in view of (8.4) and (8.5),

$$
\max \left(s_{1}^{0}, s_{2}^{0}\right) \geqslant \min \left(\max \left(E_{1}, \frac{r E_{1}}{q_{1}}\right), \max \left(E_{1}, \frac{r E_{1}}{q_{2}}\right)\right)
$$

according to $t_{0}<T$ and $\left(s_{1}^{0}, s_{2}^{0}, t_{0}\right) \in \sum\left(t_{0} ; E_{1}\right)$.

The contradiction shows that

$$
\left(s_{1}^{0}, s_{2}^{0}, t_{0}\right) \in \sum\left(t ; E_{2}\right)
$$

\section{REMARK 8.1}

$$
\sum(t ; E) \subset \sum(t ; 0) .
$$

Due to (7.34) and (8.7), we can give an evaluation figure of the optimal exercise boundary to the American call-max option.

At the 'initial time $\tau=0$ ' (expiration time $t=T$ ), the stopping region is $\sum(T ; E)$ (see (8.4)), then as $\tau>0(t<T)$ the region is separated at the ray $s_{1}=s_{2}$ into two parts, and each subregion goes to opposite side monotonical with respect to 'time $\tau$ '. One of the subregion in $\left\{s_{1}>s_{2}\right\}$ is denoted by $\sum^{(1)}(t)$ and the other in $\left\{s_{1}<s_{2}\right\}$ is denoted by $\sum^{(2)}(t)$.

THEOREM 8.3 The price $V\left(s_{1}, s_{2}, t\right)$ of American call-max option as a function of $s_{1}, s_{2}$ is convex. The stopping subregions $\sum^{(1)}(t)$ and $\sum^{(2)}(t)$ are convex sets.

Proof. The convexity of the subregion $\sum^{(i)} i=1,2$ is the consequence of the convexity of solution $V\left(s_{1}, s_{2}, t\right)$.

In fact, wlog, we assume $P\left(s_{1}, s_{2}, t\right), P_{0}\left(s_{1}^{0}, s_{2}^{0}, t\right) \in \sum^{(1)}(t)$ and

$$
P_{\lambda}:\left(s_{1}^{0}+\lambda\left(s_{1}-s_{1}^{0}\right), s_{2}^{0}+\lambda\left(s_{2}-s_{2}^{0}\right), t\right), P_{\lambda} \in \overline{P P_{0}},(0 \leqslant \lambda \leqslant 1) .
$$

According to the convexity of $V\left(s_{1}, s_{2}, t\right)$, we have

$$
0 \leqslant(1-\lambda) G_{E}\left(s_{1}^{0}, s_{2}^{0}\right)+\lambda G_{E}\left(s_{1}, s_{2}\right)-V\left(s_{1}^{0}+\lambda\left(s_{1}-s_{1}^{0}\right), s_{2}^{0}+\lambda\left(s_{2}-s_{2}^{0}\right), t\right) .
$$

In view of $P, P_{0} \in \sum^{(1)}(t), s_{1}>s_{2}, s_{1}^{0}>s_{2}^{0}$ and (8.5)

$$
s_{1}>\max \left(E, \frac{E q_{1}}{r}\right), \quad s_{1}^{0}>\max \left(E, \frac{E q_{1}}{r}\right) .
$$

So

$$
G_{E}\left(s_{1}, s_{2}\right)=s_{1}-E, \quad G_{E}\left(s_{1}^{0}, s_{2}^{0}\right)=s_{1}^{0}-E .
$$


Therefore, by virtue of (8.8),

$$
\begin{array}{r}
V\left(s_{1}^{0}+\lambda\left(s_{1}-s_{1}^{0}\right), s_{2}^{0}+\lambda\left(s_{2}-s_{2}^{0}\right), t\right) \leqslant(1-\lambda)\left(s_{1}^{0}-E\right)+\lambda\left(s_{1}-E\right) \\
=s_{1}^{0}+\lambda\left(s-s_{1}^{0}\right)-E=G_{E}\left(s_{1}^{0}+\lambda\left(s_{1}-s_{1}^{0}\right), s_{2}^{0}+\lambda\left(s_{2}-s_{2}^{0}\right)\right)
\end{array}
$$

i.e. $P \lambda \in \sum^{(1)},(0 \leqslant \lambda \leqslant 1)$.

To verify the convexity of the solution of problem (1.2)-(1.5), we consider a penalized problem (2.16), (7.17) at first and claim

$$
J\left(\hat{v} ; \alpha_{1}, \alpha_{2}\right)=\alpha_{1}^{2} \frac{\partial^{2} \hat{v}}{\partial x_{1}^{2}}+2 \alpha_{1} \alpha_{2} \frac{\partial^{2} \hat{v}}{\partial x_{1} \partial x_{2}}+\alpha_{2}^{2} \frac{\partial^{2} \hat{v}}{\partial x_{2}^{2}}-\alpha_{1}^{2} \frac{\partial \hat{v}}{\partial x_{1}}-\alpha_{2}^{2} \frac{\partial \hat{v}}{\partial x_{2}} \geqslant 0
$$

for any $\alpha_{1}, \alpha_{2} \geqslant 0$. This is a consequence of the estimate (6.5), where

$$
\frac{\bar{\sigma}_{1}}{\sigma_{1}}=\frac{\bar{\sigma}_{2}}{\sigma_{2}}=2, \alpha_{i}=\frac{3}{2} \sigma_{i} \text { and } \rho=1 \text {. }
$$

Set $\hat{V}\left(s_{1}, s_{2}, t\right)=\hat{v}\left(\ln s_{1}, \ln s_{2}, t\right)$. According to (8.9), a straight calculation shows

$$
\begin{aligned}
\frac{\partial^{2}}{\partial \tau^{2}} \hat{V}\left(s_{1}+\right. & \left.\tau \Delta s_{1}, s_{2}+\tau \Delta s_{2}, t\right)=\frac{\partial^{2}}{\partial \tau^{2}} \hat{v}\left(\ln \left(s_{1}+\tau \Delta s_{1}\right), \ln \left(s_{2}+\tau \Delta s_{2}\right), t\right) \\
= & \left(\frac{\Delta s_{1}}{s_{1}+\tau \Delta s_{1}}\right)^{2}\left(\frac{\partial^{2} \hat{v}}{\partial x_{1}^{2}}-\frac{\partial \hat{v}}{\partial x_{1}}\right)+2\left(\frac{\Delta s_{1}}{s_{1}+\tau \Delta s_{1}}\right)\left(\frac{\Delta s_{2}}{s_{2}+\tau \Delta s_{2}}\right) \frac{\partial^{2} \hat{v}}{\partial x_{1} \partial x_{2}} \\
& +\left(\frac{\Delta s_{2}}{s_{2}+\tau \Delta s_{2}}\right)^{2}\left(\frac{\partial^{2} \hat{v}}{\partial x_{2}^{2}}-\frac{\partial \hat{v}}{\partial x_{2}}\right)=J\left(\hat{v} ; \frac{\Delta s_{1}}{s_{1}+\tau \Delta s_{1}}, \frac{\Delta s_{2}}{s_{2}+\tau \Delta s_{2}}\right) \geqslant 0, \\
(1-\lambda) \hat{V}\left(s_{1}, s_{2}, t\right)+\lambda \hat{V}\left(s_{1}+\Delta s_{1}, s_{2}+\Delta s_{2}, t\right)-\hat{V}\left(s_{1}+\lambda \Delta s_{1}, s_{2}+\lambda \Delta s_{2}, t\right) & \\
= & \lambda \int_{\lambda}^{1}(1-\tau) \frac{\partial^{2}}{\partial \tau^{2}} \hat{V}\left(s_{1}+\tau \Delta s_{1}, s_{2}+\tau \Delta s_{2}, t\right) \mathrm{d} \tau \\
& +(1-\lambda) \int_{0}^{\lambda} \tau \frac{\partial^{2}}{\partial \tau^{2}} \hat{V}\left(s_{1}+\tau \Delta s_{1}, s_{2}+\tau \Delta s_{2}, t\right) \mathrm{d} \tau \geqslant 0
\end{aligned}
$$

for any $\lambda \in[0,1]$. Taking the limits as $\delta \rightarrow 0$ and $\varepsilon \rightarrow 0$, the proof of Theorem 8.3 is complete.

Finally, we study the asymptotic behaviour of the optimal exercise boundaries as $s_{1} \rightarrow \infty$ or $s_{2} \rightarrow \infty$. According to the convexity of the stopping regions and the boundary conditions (1.4) on $s_{1}=0$ and (1.5) on $s_{2}=0$, optimal exercise boundaries $\Gamma_{i}(t ; E),(i=1,2)$ can be expressed in the form

$$
\begin{array}{ll}
\Gamma_{1}(t ; E) & s_{2}=\varphi_{1}\left(s_{1}, t\right) \\
\Gamma_{2}(t ; E) & s_{1}=\varphi_{2}\left(s_{2}, t\right) .
\end{array}
$$

THEOREM 8.4 The optimal exercise boundaries $\Gamma_{i}(t ; E)(i=1,2)$ have the following properties:

(A) $\varphi_{1}\left(s_{1}, t\right)$ and $\varphi_{2}\left(s_{2}, t\right)$ are monotonically increasing in $s_{1}$ and $s_{2}$,

(B) $\varphi_{1}\left(s_{1}, t\right)$ and $\varphi_{2}\left(s_{2}, t\right)$ are concave in $s_{1}$ and $s_{2}$, 
(C)

$$
\begin{aligned}
& \lim _{s_{1} \rightarrow \infty} \frac{\varphi_{1}\left(s_{1}, t\right)}{s_{1}}=\left(\xi_{2}(t)\right)^{-1} \\
& \lim _{s_{2} \rightarrow \infty} \frac{\varphi_{2}\left(s_{2}, t\right)}{s_{2}}=\xi_{1}(t),
\end{aligned}
$$

where $\xi_{1}(t), \xi_{2}(t)$ is defined in (7.11) (7.14), $s_{1}=\xi_{i}(t) s_{2}(i=1,2)$ are optimal exercise boundaries in the case of strike price $E=0$.

\section{Proof. (A) Rescaling $s_{1}$ and $s_{2}$}

$$
s_{1}=E \tilde{s}_{1}, \text { and } s_{2}=E \tilde{s}_{2},
$$

and introducing a new function

$$
V=E \tilde{V}
$$

then we have $\tilde{V}$ as a function of $\tilde{s}_{1}$ and $\tilde{s}_{2}$ is a solution of the problem (1.2)-(1.4) with the strike price $E=1$, in which the payoff function is

$$
G\left(\tilde{s}_{1}, \tilde{s}_{2}\right)=\left(\max \left(\tilde{s}_{1}, \tilde{s}_{2}\right)-1\right)^{+} .
$$

Assume that $\tilde{\Gamma}_{1}(t): \tilde{s}_{2}=\tilde{\varphi}_{1}\left(\tilde{s}_{1}, t\right)$ and $\tilde{\Gamma}_{2}(t): \tilde{s}_{1}=\tilde{\varphi}_{2}\left(\tilde{s}_{2}, t\right)$ are optimal exercise boundaries of the American call-max problem (1.2)-(1.4) with $E=1$. Then we have

$$
\varphi_{i}\left(s_{i}, t\right)=E \tilde{\varphi}_{i}\left(\frac{s_{i}}{E}, t\right) \quad(i=1,2) .
$$

Therefore we only need to prove the theorem in the case of $E=1$. Due to (8.6), if $E_{1}>E_{2}$,

$$
E_{2} \tilde{\varphi}_{i}\left(\frac{s_{i}}{E_{2}}, t\right)>E_{1} \tilde{\varphi}_{i}\left(\frac{s_{i}}{E_{1}}, t\right) \quad(i=1,2)
$$

i.e. if $\frac{s_{i}}{E_{2}}>\frac{s_{i}}{E_{1}}$, for $s_{i}>0$,

$$
\tilde{\varphi}_{i}\left(\frac{s_{i}}{E_{2}}, t\right)>\frac{E_{1}}{E_{2}} \tilde{\varphi}_{i}\left(\frac{s_{i}}{E_{1}}, t\right)>\tilde{\varphi}_{i}\left(\frac{s_{i}}{E_{1}}, t\right) \quad(i=1,2) .
$$

So $\tilde{\varphi}_{i}\left(s_{i}, t\right)$ is monotonically increasing in $s_{i}$.

(B) is a consequence of Theorem 8.3.

Now we turn to prove the property (C). We know (see (3.6)) that

$$
\lim _{E \rightarrow 0} V\left(s_{1}, s_{2}, t ; E\right)=V_{0}\left(s_{1}, s_{2}, t\right)
$$

where $V_{0}$ is a solution of problem (7.1)-(7.4). As a consequence, we have

$$
\lim _{E \rightarrow 0} \varphi_{i}\left(s_{i}, t\right)=\varphi_{i}^{0}\left(s_{i}, t\right), \quad(i=1,2)
$$

where $\Gamma_{1}(t ; 0): s_{2}=\varphi_{1}^{0}\left(s_{1}, t\right)$ and $\Gamma_{2}(t ; 0): s_{1}=\varphi_{2}^{0}\left(s_{2}, t\right)$. In view of (8.13) for fixed $s_{i}, s_{i}=E \tilde{s}_{i}$,

$$
\lim _{\tilde{s}_{i} \rightarrow \infty} \frac{\tilde{\varphi}_{i}\left(\tilde{s}_{i}, t\right)}{\tilde{s}_{i}}=\lim _{E \rightarrow 0} \frac{E}{s_{i}} \tilde{\varphi}_{i}\left(\frac{s_{i}}{E}, t\right)=\lim _{E \rightarrow 0} \frac{\varphi_{i}\left(s_{i}, t\right)}{s_{i}}=\frac{\varphi_{i}^{0}\left(s_{i}, t\right)}{s_{i}}= \begin{cases}\left(\xi_{2}(t)\right)^{-1} & i=1 \\ \xi_{1}(t) & i=2\end{cases}
$$

according to (7.34).

The proof of Theorem 8.4 is complete. 


\section{Conclusions}

In accordance with the results mentioned above, even if we have not got an explicit form of the solution, we still can draw a figure of the optimal exercise boundary to the American max-call option as follows:

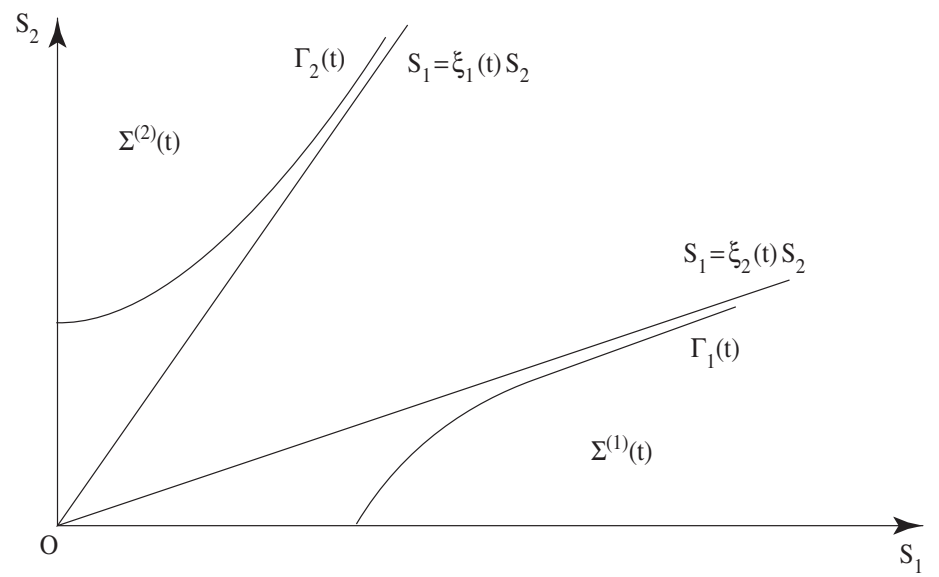

\section{Acknowledgements}

The work has been supported by the National Foundation of China. The author would like to thank the referee for pointing out the recent work [10], in which the non-emptiness and the shape, especially the asymptotic behaviour near the expiry date of the stopping region, of American options on several assets are studied.

\section{REFERENCES}

1. Broadie, M. \& Detemple, J. The valuation of American options on multiple assets. Math. Finance 7, (1997) 241-286.

2. STUlz, R. M. Options on the minimum or the maximum of two risky assets. J. Financial Economics 10, (1982) 161-185.

3. Johnson, H. Options on the maximum or the minimum of several assets. J. Financial Quantitative Analysis 22, (1987) 227-283.

4. Hull, J. C. Options, Futures and their Derivatives, 3rd edn. Prentice-Hall, Englewood Cliffs, NJ (1997).

5. Kinderlehrer, D. \& Stampacchia, G. An Introduction to Variational Inequalities and their Applications. Academic Press, New York (1980).

6. Friedman, A. Variational Principles and Free Boundary Problems. Wiley-Interscience, New York (1982).

7. Dewynne, J. N., Howison, S. D., Rupf, I., \& Wilmott, P. Some mathematical results in the pricing of American options. Eur. J. Appl. Math. 4, (1993) 381-398.

8. MARgRALE, W. The value of an option to exchange one asset for another. J. Finance 33, (1978) 177-186.

9. Rubinstein, M. One for another. Risk (1991).

10. Villeneuve, S. Exercise regions of American options on several assets. Finance and Stochastics 3, (1999) 295-322. 